\title{
Adipose-derived stem cells versus bone marrow-derived stem cells for vocal fold regeneration.
}

\section{$\operatorname{AUTHOR(S):~}$}

Hiwatashi, Nao; Hirano, Shigeru; Mizuta, Masanobu; Tateya, Ichiro; Kanemaru, Shin-ichi; Nakamura, Tatsuo; Ito, Juichi

\section{CITATION:}

Hiwatashi, Nao ... [et al]. Adipose-derived stem cells versus bone marrow-derived stem cells for vocal fold regeneration.. The Laryngoscope 2014, 124(12): E461-E469

\section{ISSUE DATE:}

2014-07-10

\section{URL:}

http://hdl.handle.net/2433/200625

\section{RIGHT:}

This is the peer reviewed version of the following article: Hiwatashi, N., Hirano, S., Mizuta, M., Tateya, I., Kanemaru, S.-i. Nakamura, T. and Ito, J. (2014), Adipose-derived stem cells versus bone marrow-derived stem cells for vocal fold regeneration. The Laryngoscope, 124: E461-E469, which has been published in final form

athttp://dx.doi.org/10.1002/lary.24816. This article may be used for non-commercial purposes in accordance with wiley Terms and Conditions for Self-Archiving.; This is not the published version. Please cite only the published version.; $こ の$ 論文は出版社版でありません。引用の際には出版社版をご確認ご利用ください。 
Adipose-derived stem cells vs. bone marrow-derived stem cells for vocal fold regeneration

(Abbreviation: Comparison of ASC and BMSC)

Nao Hiwatashi ${ }^{1}$, MD

Shigeru Hirano ${ }^{1}$, MD, PhD

Masanobu Mizuta², 1, MD

Ichiro Tateya ${ }^{1}, \mathrm{MD}, \mathrm{PhD}$

Shin-ichi Kanemaru ${ }^{3,4,1}$, MD, PhD

Tatsuo Nakamura ${ }^{5}, \mathrm{MD}, \mathrm{PhD}$

Juichi Ito ${ }^{1}$, MD, PhD

${ }^{1}$ Department of Otolaryngology-Head and Neck Surgery, Graduate School of Medicine, Kyoto

University, Kyoto

${ }^{2}$ Department of Otolaryngology, Vanderbilt University, Nashville, TN

${ }^{3}$ Department of Otolaryngology, Department of Regenerative Treatment for Tympanic Membrane, the Foundation for Biomedical Research and Innovation

${ }^{4}$ Department of Otolaryngology-Head and Neck Surgery, Kitano Hospital, Tazuke Kofukai Medical Research Institute, Osaka

${ }^{5}$ Department of Bioartificial Organs, Institute for Frontier Medical Science, Kyoto University, Kyoto 
$\checkmark \quad$ This study was supported by the Advanced Research for Medical Products

Mining Program of the National Institute of Biomedical Innovation (NIBIO).

$\checkmark \quad$ Presented at the 135th American Laryngological Association, Las Vegas, Nevada, U.S.A., May 14 - 18, 2014.

$\checkmark \quad$ No financial disclosure.

$\checkmark$ Conflict of Interest: None

\section{Correspondence to:}

Shigeru Hirano, MD, PhD, Department of Otolaryngology-Head \& Neck Surgery, Graduate School of Medicine, Kyoto University, Sakyo-ku, Kyoto 606-8507, Japan.

Tel: +81-75-751-3346, Fax: +81-75-751-7225, E-mail: hirano@ent.kuhp.kyoto-u.ac.jp 


\section{Abstract}

Objectives: Vocal fold scarring presents therapeutic challenges. Recently, cell therapy with mesenchymal stromal cells (MSC) has become a promising approach. The aim of this study was to compare the therapeutic potential of adipose-derived stem cells (ASC) with bone marrow-derived stem cells (BMSC) for vocal fold regeneration.

Study Design: Prospective animal experiments with controls.

Methods: The vocal folds of Sprague-Dawley rats were unilaterally injured. Two months after injury, rats were treated with a local injection of ASC (ASC group), BMSC (BMSC group) or saline (sham-treated group). The GFP-labeled ASC and BMSC were extracted from CAG-EGFP rats. Larynges were harvested for histological and immunohistochemical examinations 1 and 3 months post-transplantation and for quantitative real-time polymerase chain reaction (PCR) 1 month post-transplantation.

Results: After 1 month, no surviving cells from the transplant were detected. Histological examination showed significantly increased hyaluronic acid (HA) and decreased dense collagen deposition in both ASC and BMSC groups compared to shams 1 and 3 months after treatment. Real-time PCR revealed that hyaluronan synthase 1 (Has1) and Has2 were upregulated in only the ASC group compared with the sham-treated group. Fibroblast growth factor 2 (basic) (Fgf2), hepatocyte growth factor $(\mathrm{Hgf})$ and Has3 were upregulated in both cell transplantation groups. ASC seemed to upregulate $\mathrm{Hgf}$ more than did BMSC.

Conclusions: The regenerative effects of ASC and BMSC transplantation were found to be similar for the restoration. It is suggested that ASC might have more potential because of better recovery of $\mathrm{HA}$, a superior anti-fibrotic effect, and the upregulation of $\mathrm{Hgf}$. 
Key Words: mesenchymal stromal cell, vocal fold scarring, adipose tissue, bone marrow, wound healing, extracellular matrix

Level of Evidence: N/A. 


\section{Introduction}

Vocal fold scarring can result from injury, inflammation or surgical treatment. Vocal folds have multi-layered structures, including the epithelium, the superficial lamina propria (SLP), and the vocal ligament. ${ }^{1}$ The SLP contains a substantial amount of hyaluronic acid (HA) and its pliability is indispensable for the vibration of normal vocal folds and phonation. Vocal fold scarring disrupts the layered structures. The changes are characterized by alterations in the organization and distribution of extracellular matrix (ECM) components, such as the deposition of disorganized collagen and elastin bundles and the loss of HA. ${ }^{2}$ These histological changes stiffen the viscoelastic properties of vocal fold mucosa and they lead to a dysphonic, often breathy and barely sustainable voice and severely impact the quality of life. ${ }^{3}$

Many therapeutic strategies have been attempted for treatment of vocal fold scarring. Although most of the current approaches such as collagen gel ingection, ${ }^{4}$ fat implants ${ }^{5}$ and fascia implants $^{6}$ aim at the augmentation or softening of the scarred SLP, the degree of effectiveness varies between individuals and these approaches do not restore a normal ECM distribution. Recovery of the pliability of vocal fold and vocal function requires regulation and restoration of ECM components. Because of the difficulty of replacing scar tissue with innate ECM, it remains a therapeutic challenge in treating vocal fold scarring. ${ }^{7}$

In recent years, stem cell-based regenerative and reparative therapies have gained significant attention in basic and clinical research. Mesenchymal stromal cells (MSC) have received much attention as a promising cell population ${ }^{8}$ because they are multipotential and capable of differentiating into bone, adipose, cartilage, and myelosupportive stroma in vitro and in vivo. ${ }^{8-10}$ MSC can be derived from bone marrow (BMSC). Kanemaru et al. initially reported that injection of BMSC in atelocollagen gel into canine injured vocal folds improved wound healing of the vocal 
folds in endoscopic and histological findings. ${ }^{11}$ In addition, BMSC are not immunogenic and could in fact be immunosuppressive. ${ }^{12}$ Although the mechanisms of immunomodulating capacity are not fully known, even xenograft transplantation using human BMSC have been reported to improve the viscoelastic and histologic properties of scarred vocal folds and/or prevented vocal fold scar formation. ${ }^{13,14}$ Moreover, MSC can sense inflammatory signals through the expression of cytokine/chemokine receptors and integrins, and migrate to sites of inflammation. ${ }^{15}$ It has been proven that systemically administered BMSC home to and reside at the site of injured vocal folds, leading to the restoration of ECM components. ${ }^{16}$

Recent studies have reported that adult adipose tissue also contains multipotent cells and that these adipose-derived stem cells (ASC) have the potential for the prevention of scar formation or the regeneration of injured vocal folds. ${ }^{17,18}$ Lee et al. demonstrated that ASC mixed with atelocollagen improved the laryngoscopic findings 6 months after initial damage. ${ }^{16}$ The advantage of ASC over BMSC is the high abundance of the stem cell population in the fat and the ease of collection. It was also reported that ASC secrete multiple factors including some strong anti-fibrotic molecules such as hepatocyte growth factor (HGF) ${ }^{19}$ However, it has not been clarified whether ASC or BMSC are better suited for vocal fold regeneration. In the current study, we compared the potentials of ASC and BMSC for treatment of chronic vocal fold scarring, focusing on histological regeneration and the gene expression of cells in SLP. 


\section{Material and Methods}

\section{Animals}

In this study, we used Sprague-Dawley (SD) rats (55 males [13-weeks-old], 10 males [25-weeks-old] and 5 males [33-weeks-old]). We also used 8 male 13-week-old GFP transgenic Sprague-Dawley rats [SD-Tg(CAG-EGFP)]. All rats were purchased from Japan SLC, Inc. (Shizuoka, Japan). Animal care was maintained in the Institute of Laboratory Animals of the Graduate School of Medicine, Kyoto University. The number of animals used in this study was kept to a minimum. All possible efforts to reduce their suffering were made in compliance with protocols established by the Animal Research Committee of Kyoto University.

\section{Isolation and Cultivation of ASCs and BMSCs}

GFP transgenic rats were separated in two groups: one was for isolation of ASC $(n=4)$ and the other was for BMSC $(n=4)$. Rats were humanely euthanized by intra-cardiac injection of pentobarbital sodium (100 mg per animal; Dainippon Pharmaceutical Co. Ltd, Osaka, Japan) after inhalative sedation with diethyl ether. For isolation of ASC, rats’ inguinal fat pads were minced and digested in a lactated Ringer solution containing $0.035 \mathrm{mg} / \mathrm{mL}$ Liberase $^{\mathrm{TM}}$, Research Grade (Roche Applied Science, Mannheim, Germany) under agitation for $40 \mathrm{~min}$ at $37^{\circ} \mathrm{C}$ and then rested for 5 min to separate the stromal cell fraction from adipocytes. The stromal cell fraction was filtered through a $100 \mu \mathrm{m}$ cell strainer (BD Biosciences, San Jose, CA) and centrifuged at $500 \mathrm{x}$ g for 5 min. The cell pellet was resuspended in a lactated Ringer solution and then filtered through a $40 \mu \mathrm{m}$ cell strainer (BD Biosciences). Isolated cells were seeded in flasks with Dulbecco’s modified Eagle's medium (Gibco, Invitrogen Co., Carlsbad, CA) containing 10\% fetal bovine serum and antibiotics. For 
isolation of BMSC, after humanely euthanizing the animals, bilateral femurs were flushed to obtain bone marrow and the cells were seeded in flasks with Dulbecco's modified Eagle's medium containing $10 \%$ fetal bovine serum and antibiotics.

The following procedure was the same for ASC and BMSC cultivation. Cells were incubated in $5 \% \mathrm{CO}_{2}$ at $37^{\circ} \mathrm{C}$ for 2 days to allow them to adhere. The medium was changed once daily until the cells reached confluence. Nonadherent cells were removed during the medium change, leaving behind only adherent ASC and BMSC. After 2 passages, the cells were collected and analyzed using a rat MSC functional identification kit (R\&D Systems, Minneapolis, MN), confirming their differentiation into adipocytes, osteocytes and chondrocytes. Third- or fourth-passage cells were used in the following experiments. Just before transplantation, cells were collected and diluted to $1.0 \times 10^{7}$ cells/mL with phosphate-buffered saline (PBS).

\section{Surgical Procedure}

The SD rats were anesthetized with an intraperitoneal injection of ketamine hydrochloride (45 $\mathrm{mg} / \mathrm{kg}$ ) and xylazine hydrochloride $(4.5 \mathrm{mg} / \mathrm{kg})$ after sedation with diethyl ether. Atropine sulfate $(0.005 \mathrm{mg} / \mathrm{kg})$ was also injected intraperitoneally to reduce the secretion of saliva and sputum in the laryngeal lumen. The animals were placed on a custom-made operating platform in a near-vertical position. The larynx was visualized with a rigid endoscope inserted transorally at an angle of $30^{\circ}$. The right vocal folds were injured by stripping with microscissors until the thyroarytenoid muscle was exposed. This surgical procedure was described previously. ${ }^{20}$ Two months after injury (animals were 21 weeks old), rats were again generally anesthetized as described above. After placement on the operating platform, a $50 \mu \mathrm{L}$ of solution containing $5.0 \times 10^{5}$ GFP-labeled ASC or BMSC was gently injected into the right thyroarytenoid muscle adjacent to the vocal fold via a 30-gauge needle affixed to a microsyringe (ASC group; $n=20$, BMSC group; $n=20$ ). The remaining injured rats 
were injected with saline (sham-treated group; $\mathrm{n}=15$ ). This time point of transplantation was selected according to the previous report that demonstrated that 2 months were required for maturation of the vocal fold scar in rats. ${ }^{20}$

The animals were euthanized as described above and each larynx was immediately harvested at the designated time points. The following assays were conducted. To assess immunohistochemical and histological changes, the ASC group and the BMSC group were sampled at time zero ( $\mathrm{n}=5$ each), 1 month $(\mathrm{n}=5$ each) and 3 months $(\mathrm{n}=5$ each). The sham-treated group was assessed at 1 month $(n=5)$ and 3 months $(n=5)$. For gene expression analysis, the ASC group, BMSC group and the sham-treated group were analyzed after 1 month ( $\mathrm{n}=5$ each). Five age-matched 25-week-old SD rats served as controls for gene expression analyses. The remaining 5 age-matched 25-week-old and 33-week-old SD rats served as controls for histological examination. These assessments were performed in a blinded fashion, in which the examiners were not informed that which rats were divided into ASC, BMSC or sham-treated group, and also into histological or gene expression analysis before the injection procedures.

\section{Immunohistochemical and histological examination}

The harvested larynges were fixed in $4 \%$ paraformaldehyde for $3 \mathrm{~h}$, then in 30\% sucrose for $24 \mathrm{~h}$ at $4^{\circ} \mathrm{C}$. After embedding in Optimum Cutting Temperature (O.C.T) formulation (Tissue-Tek, Kyoto, Japan), the samples were frozen and 10 micrometer-thick serial coronal sections were placed on microscope slides.

We performed immunohistochemical analysis to evaluate technical variations and the survival of injected cells. Sections were incubated in primary rabbit monoclonal antibody against GFP (1:200; MBL International Corp., Woburn, MA) overnight at room temperature. The samples were washed and incubated for $1 \mathrm{~h}$ at room temperature, and Alexa-Flour 488 anti-rabbit IgG (1:500; 
Invitrogen Co., Carlsbad, CA) was used as the secondary antibody. Stained sections were visualized and images were captured using a BIOREVO BZ-9000 microscope (Keyence Co., Osaka, Japan). All images were captured at 4 - 10x magnification and the number of GFP-positive cells was counted from 3 different sections: anterior, middle, and posterior portions. To assess the groups that underwent transplantation under the same conditions at the indicated times, we compared the results to those obtained with the ASC group and BMSC group that were sacrificed immediately after transplantation (month 0). The number of GFP-positive cells was totaled in the 3 sections and the average was calculated. The distance between the lateral edge of the LP and the most adjacent transplanted cells was measured in each section and the average was also calculated.

We performed histological analyses to evaluate changes in the ECM or the degree of scar contraction of scarred vocal folds. Alcian blue staining and a hyaluronidase digestion technique were performed to identify HA, whereas Elastica van Gieson staining was used to measure collagen abundance. Images were also captured at 10x magnification with a BIOREVO BZ-9000 microscope. To evaluate the density of the HA or collagen area in each LP, we totaled the areas stained for HA or collagen in the injured right LP from the 3 sections and they were divided by the totaled whole LP areas. The evaluation of the density of HA or collagen area was conducted not only for the treatment side (right side) but the contralateral side (normal left side) as internal controls. The thickness of the LP was also evaluated by measuring the distances from the free edge of the vocal fold down to the thyroarytenoid muscle. The distances were totaled and normalized by dividing the thickness of LP of the normal side.

BIOREVO BZ-H1C and BZ-H1M software (Keyence Co.) were used to count the cells, and to determine areas and distances. All images were captured in the same way. These assessments were performed in a blinded fashion, in which the examiners were not informed of the group to which each slide belonged. 


\section{Gene Expression Analysis}

Quantitative real-time polymerase chain reaction (qRT-PCR) was performed to examine the changes in mRNA expression of ECM components and endogenous growth factors induced by cell transplantation. Total RNA was extracted from the epithelium and LP of the right vocal fold with Trizol Reagent (Invitrogen Co.) following the manufacturer's protocol. Extracted total RNA was quantified and qualified by measuring the A260/280 ratio of each sample. Reverse transcription was performed by using a High Capacity cDNA Reverse Transcription Kit (Applied Biosystems, Foster City, CA). Reactions were performed with the GeneAmp polymerase chain reaction (PCR) system 9700 (Applied Biosystems) with the following parameters: $25^{\circ} \mathrm{C} /$ ten $\mathrm{min}, 48^{\circ} \mathrm{C} / 30 \mathrm{~min}, 95^{\circ} \mathrm{C} / 5 \mathrm{~min}$ and $4^{\circ} \mathrm{C} / 5 \mathrm{~min}$.

We examined the mRNA expression of endogenous fibroblast growth factor 2 (basic) (Fgf2), hepatocyte growth factor (Hgf), vascular endothelial growth factor type A (Vegfa), hyaluronan synthase 1 (Has1), Has2 and Has3, procollagen types I (Col1a1) and III (Col3a1), matrix metalloproteinase (Mmp1) and Mmp8. The primer sequences are listed in the Table. Amplification was performed in a $20 \mu \mathrm{L}$ reaction mixture containing $1 \mu \mathrm{L}$ of cDNA, $10 \mu \mathrm{L}$ of Power SYBR Green Master Mix (Applied Biosystems), $0.25 \mu \mathrm{mol} / \mathrm{L}$ final concentration of each primer, and ribonuclease-free water under the following conditions: $95^{\circ} \mathrm{C} / 10 \mathrm{~min}$ and 40 cycles of $95^{\circ} \mathrm{C} / 15 \mathrm{~s}$ and $60^{\circ} \mathrm{C} / 1$ min. Fluorescence was detected with StepOne Plus (Applied Biosystems). The specificity of each primer was confirmed by a single peak melting curve. From ASCs, BMSCs, sham group cells (1 month after cell transplantation or saline injection) and age-matched 25-week-old SD rats (normal control), 5 samples each were assayed and tested in triplicate. Standard curves were used and results are shown by the fold-change relative to the control target gene mRNA concentration normalized to the housekeeping gene, $\beta 2$-microglobulin mRNA. 
Statistical Analysis

Statistical analyses were performed using one-way factorial analysis of variance (ANOVA) followed by a Tukey-Kramer post hoc test for the investigation of HA and collagen ratio, the thickness of LP and qRT-PCR. A Student $t$ test was used to investigate the analysis of technical variations. Statistical significance was defined as $p<0.01$ or $p<0.05$ using StatView 5.0 (SAS Institute, Berkeley, CA). All data are expressed as the means \pm standard deviation. 


\section{RESULTS}

\section{Placement of transplanted ASC and BMSC}

To confirm the location of the transplanted cells and to demonstrate the accuracy of the injection procedure, we examined the placement of the injected cells just after the transplantation (month zero). Most of the GFP-positive transplanted cells were located within the outer space of the thyroarytenoid muscle (Fig. 1A). There were no distinct differences between the ASC group and the BMSC group in terms of the average number of cells (Fig. 1B). The average distances between the lateral edge of the LP and the closest transplanted cells also showed no significant differences between the 2 groups (Fig. 1C). Both cell numbers and distances showed normal distributions with the F-test conducted prior to the Student $t$ test.

\section{Survival of Transplanted Cells}

At 1 month post-transplantation, no GFP-positive cells were detected among any sections of either the ASC or the BMSC group (Fig. 1A). Similarly, no cells were found to migrate or distribute in either the LP or underlying muscles 3 months post-transplantation.

\section{Restoration of Scarred Vocal Folds}

HA was detected by Alcian blue staining. The blue stain decolorized following hyaluronidase digestion. The sham-treated group showed significantly less HA, whereas in both the ASC and BMSC groups, HA was retained to a certain degree (Fig. 2A). The restoration of HA appeared to be more augmented at the anterior portions than the middle or posterior portions. The image analyses revealed that the density of HA-stained areas was significantly greater in ASC and BMSC groups 
than in the sham-treated group, whereas there was no significant difference between the 2 transplantation groups (Fig. 2B, 2C). The significant HA restorations appeared at 1 and 3 months post-transplantation. With Elastica van Gieson staining, severe contraction and dense collagen deposition were observed in the LP of the sham-treated group. On the contrary, these undesirable histological changes were relatively mild in the ASC and BMSC groups (Fig. 3A). Compared with the sham-treated group, the density of collagen-rich areas was significantly decreased in ASC and BMSC group 1 and 3 month post-transplantation (Fig. 3B, 3C). There was no significant difference between the 2 transplantation groups. For internal controls, we also calculated the density of HA and collagen of the contralateral side, and there were no significant differences between each group (Fig 4A, 4B). The thickness of the LP was significantly greater in both ASC and BMSC group 1 and 3 months post-transplantation compared with the sham-treated group (Fig. 5). The ratio of HA increase, collagen reduction, and the thickness of LP appeared to be slightly enhanced as time passed. No remarkable change was observed in the underlying muscles.

\section{Gene expression analyses}

Endogenous Fgf2 and Hgf demonstrated similar expression levels that were significantly higher in both the ASC and BMSC groups than in the sham-treated group, whereas the ASC group showed more upregulation than the BMSC group (Fig. 6).

As for ECM gene expression analyses, only the ASC group significantly upregulated the expression of Has1 and Has2 compared with the sham-treated group. Has3 was upregulated in both the ASC and BMSC groups. Col3a1 expression was significantly higher in the BMSC group than the sham-treated groups. Although the expression of Col1a1, Mmp1, Mmp8, and Vegfa was slightly upregulated in the 2 cell transplantation groups, there were no significant differences between the groups. 


\section{Discussion}

In recent years, stem cells have received widespread attention in the fields of regenerative and reparative medicine, including the use of embryonic stem (ES) cells and induced pluripotent stem (iPS) cells. Both cell types are promising tools and could be candidates for regeneration of injured or diseased organs. However, there remain significant problems for clinical application, such as ethical issues and tumorigenicity, though efforts are being focused on finding solutions. On the other hand, autologous MSC are considered to be a more promising tool as they can avoid the ethical issues and have already been applied widely in regenerative medicine. MSC were first identified in bone marrow, ${ }^{21}$ and likely reside in other tissues of mesodermal origin: adipose tissue, umbilical cord, placenta, and dental pulp. ${ }^{22-25}$ Among these tissues, adipose tissue has the advantage of containing at least a thousand-fold more stem cells than does marrow ${ }^{26}$. Another advantage is easier accessibility. ASC are reported to have a gene expression profile similar to BMSC. ${ }^{27}$

In the current study, we transplanted allogeneic MSC. In terms of immune rejection, autologous MSC might be ideal materials. However, the cell extracting procedures might be stressful for rats and might have some unfavorable effects on wound healing of vocal folds. We used allogeneic MSC based on the fact that MSC are reported to express constitutively low levels of cell-surface HLA class I molecules and lack expression of HLA class II leading to the absence or reduced activation of innate and adaptive immune responses, thus avoiding rejection of the allogeneic MSC. ${ }^{28}$ To identify cells, nuclear staining can be used with GFP-labeled cells to investigate cell survival; however, the staining dilutes out during cell proliferation. In our current results, the locally injected cells were not detected either in SLP or in the underlying muscles 1 month post-transplantation. Some investigators previously reported that transplanted MSC survive in the injured vocal folds or its underlying muscles for 1 or 2 months post-transplantation. ${ }^{11,17,18}$ 
Kanemaru et al. also demonstrated that transplanted MSC differentiated into at least 2 lineages, epithelial cells and muscle. ${ }^{29}$ The differences observed in the current study might be due to the earlier use of a scaffold as the carrier of MSC. In this study, we simply injected cells without any scaffolds in order to clarify the effects achieved with a pure cell suspension.

The histological examination demonstrated restoration of HA and reduction of collagen deposition in both the ASC and BMSC groups compared with the sham treated group. HA plays a beneficial role in maintaining ideal viscoelastic function of vocal folds, ${ }^{30}$ and increased collagen content in SLP correlates to the stiffness that disturbs the pliable mucosal wave. ${ }^{31}$ Such ECM repair might improve the mucosal wave of vibration and glottal competence. Moreover, as we showed, ECM restoration subsequently augmented the thickness of the SLP that would improve the glottal gap. In most of the specimens, the thickness of SLP seemed to be larger in the anterior portion than middle or posterior portion. That might come from the technical limitation because anterior portion of SLP is difficult to be totally resected to avoid the web formation after surgery. Apart from the effect on the SLP, there was no remarkable change in the underlying muscle. One reason may be due to the difference of intensity of injury. As we injected the cells gently, the injury that occurred in the muscle was relatively minor, therefore any remarkable changes such as contraction or augmentation were not shown.

Although there were no significant differences in these histological findings between the ASC and BMSC groups, gene expression analysis revealed that only the ASC groups showed a significant upregulation of Has1 and Has2 compared to sham. As the upreguration of Has is closely related with the production of HA, the results indicated that ASC might have more potential for HA restoration. On the contrary, the expression of Col3a1 showed significant upregulation in the BMSC group compared to the sham-treated group. Collagen type III acts as a temporary scaffold converting to collagen type I during the wound healing process which provides long-term tensile strength to the 
wound site and its excessive accumulation leads to a loss of vocal fold viscoelasticity. The expression of Mmp which reflects the collagen turnover process in tissues showed a tendency to increase in BMSC groups. Thus, BMSC might have more potential in the turnover of collagen.

With regard to growth factors, both gene expression of Fgf2 and Hgf were significantly upregulated in both ASC and BMSC groups. The effects on Hgf seemed to be stronger in the ASC group than the BMSC group (ASC group, $\mathrm{p}<0.01$; BMSC group, $\mathrm{p}<0.05$, compared with the sham-treated group). HGF has a strong anti-fibrotic effect and it has proven to contributes to the prevention or complete resolution of fibrosis and improves the regeneration of some organs such as liver, kidney, lung, etc. ${ }^{32}$ In an in vitro model, Kumai et al. demonstrated increased HGF secretion from ASC when co-cultured with scarred vocal fold fibroblasts. ${ }^{33}$ HGF was also revealed to be effective for the treatment of chronic vocal fold scar. ${ }^{34}$ FGF2 has anti-fibrotic effects in itself and also stimulates the vocal fold fibroblasts to upregulate gene expression of $\mathrm{Hgf} \cdot{ }^{35}$ In the current study, considering that the injected ASC and BMSC were found to be placed neither in the SLP nor the TA muscle, these factors secreted from injected cells should have affected the fibroblasts of scarred vocal folds while the injected cells have survived, and subsequently leads to the continuous histological restoration and upregulation of gene expression even after the injected cells have totally disappeared. These paracrine effects were thought to be beneficial for vocal fold regeneration.

The current results indicate that ASC and BMSC have similar effects on regeneration of the vocal folds in terms of reconstitution of the ECM in the SLP. It is also suggested that ASC might have an advantage in upregulating greater secretion of HGF and HAS, which might lead to better regeneration of the vocal fold compared to BMSC.

In our study, there is a limitation that the number of samples were small to exclude statistical issue such as type II error. Also, the observation period may not long enough in terms of the duration of regeneration and the safety aspect. Functional examinations are also required to 
assess viscoelasticity of the treated vocal folds. 


\section{Conclusions}

The current study compared regenerative effects of ASC and BMSC for scarred vocal folds. The results have demonstrated similar histological regeneration in both groups, while ASC was more effective at upregulating gene expression of Has1, Has2 and Hgf, which might indicate greater potential of ASC for HA production and anti-fibrotic effects. 


\section{Acknowledgements}

This study was supported by the Advanced Research for Medical Products Mining Program of the National Institute of Biomedical Innovation. 


\section{References}

1. Gray SD, Titze IR, Alipour F, Hammond TH. Biomechanical and histologic observations of vocal fold fibrous proteins. Ann Otol Rhinol Laryngol 2000; 109:77-85.

2. Hirano S, Minamiguchi S, Yamashita M, Ohno T, Kanemaru S, Kitamura M. Histologic characterization of human scarred vocal folds. J Voice 2009; 23:399-407.

3. Friedrich G, Dikkers FG, Arens Cet al. Vocal fold scars: current concepts and future directions. Consensus report of the Phonosurgery Committee of the European Laryngological Society. Eur Arch Otorhinolaryngol 2013; 270:2491-2507.

4. Remacle M, Lawson G, Degols JC, Evrard I, Jamart J. Microsurgery of sulcus vergeture with carbon dioxide laser and injectable collagen. Ann Otol Rhinol Laryngol 2000; 109:141-148.

5. Sataloff RT, Spiegel JR, Hawkshaw M, Rosen DC, Heuer RJ. Autologous fat implantation for vocal fold scar: a preliminary report. J Voice 1997; 11:238-246.

6. Tsunoda K, Kondou K, Kaga Ket al. Autologous transplantation of fascia into the vocal fold: long-term result of type-1 transplantation and the future. Laryngoscope 2005; 115:1-10.

7. McGee GS, Davidson JM, Buckley Aet al. Recombinant basic fibroblast growth factor accelerates wound healing. J Surg Res 1988; 45:145-153.

8. Pittenger MF, Mackay AM, Beck SCet al. Multilineage potential of adult human mesenchymal stem cells. Science 1999; 284:143-147.

9. Haynesworth SE, Goshima J, Goldberg VM, Caplan AI. Characterization of cells with osteogenic potential from human marrow. Bone 1992; 13:81-88.

10. Liechty KW, MacKenzie TC, Shaaban AFet al. Human mesenchymal stem cells engraft and demonstrate site-specific differentiation after in utero transplantation in sheep. Nat Med 2000; 6:1282-1286.

11. Kanemaru SI, Nakamura T, Omori Ket al. Regeneration of the vocal fold using autologous mesenchymal stem cells. Annals of Otology Rhinology and Laryngology $2003 ; 112$.

12. Le Blanc K. Immunomodulatory effects of fetal and adult mesenchymal stem cells. Cytotherapy 2003; 5:485-489.

13. Hertegård S, Cedervall J, Svensson Bet al. Viscoelastic and histologic properties in scarred rabbit vocal folds after mesenchymal stem cell injection. Laryngoscope 2006; 
116:1248-1254.

14. Svensson B, Nagubothu SR, Cedervall Jet al. Injection of human mesenchymal stem cells improves healing of vocal folds after scar excision--a xenograft analysis. Laryngoscope 2011; 121:2185-2190.

15. Yagi H, Soto-Gutierrez A, Parekkadan Bet al. Mesenchymal stem cells: Mechanisms of immunomodulation and homing. Cell Transplant 2010; 19:667-679.

16. Ohno S, Hirano S, Kanemaru Set al. Role of circulating MSCs in vocal fold wound healing. Laryngoscope 2012; 122:2503-2510.

17. Lee BJ, Wang SG, Lee JCet al. The prevention of vocal fold scarring using autologous adipose tissue-derived stromal cells. Cells Tissues Organs 2006; 184:198-204.

18. $\mathrm{Xu} \mathrm{W}, \mathrm{Hu} \mathrm{R}$, Fan E, Han D. Adipose-derived mesenchymal stem cells in collagen-hyaluronic acid gel composite scaffolds for vocal fold regeneration. Ann Otol Rhinol Laryngol 2011; 120:123-130.

19. Rehman J, Traktuev D, Li Jet al. Secretion of angiogenic and antiapoptotic factors by human adipose stromal cells. Circulation 2004; 109:1292-1298.

20. Tateya T, Tateya I, Sohn JH, Bless DM. Histologic characterization of rat vocal fold scarring. Annals of Otology Rhinology and Laryngology 2005; 114:183-191.

21. Friedenstein AJ, Gorskaja JF, Kulagina NN. Fibroblast precursors in normal and irradiated mouse hematopoietic organs. Exp Hematol 1976; 4:267-274.

22. Zuk PA, Zhu M, Ashjian Pet al. Human adipose tissue is a source of multipotent stem cells. Mol Biol Cell 2002; 13:4279-4295.

23. Romanov YA, Svintsitskaya VA, Smirnov VN. Searching for alternative sources of postnatal human mesenchymal stem cells: candidate MSC-like cells from umbilical cord. Stem Cells 2003; 21:105-110.

24. Fukuchi Y, Nakajima H, Sugiyama D, Hirose I, Kitamura T, Tsuji K. Human placenta-derived cells have mesenchymal stem/progenitor cell potential. Stem Cells 2004; 22:649-658.

25. Gronthos S, Mankani M, Brahim J, Robey PG, Shi S. Postnatal human dental pulp stem cells (DPSCs) in vitro and in vivo. Proc Natl Acad Sci U S A 2000; 97:13625-13630.

26. Fraser JK, Wulur I, Alfonso Z, Hedrick MH. Fat tissue: an underappreciated source of stem cells for biotechnology. Trends Biotechnol 2006; 24:150-154.

27. Lee RH, Kim B, Choi Iet al. Characterization and expression analysis of mesenchymal stem cells from human bone marrow and adipose tissue. Cell Physiol Biochem 2004; 14:311-324. 
28. Kahan BD. Individuality: the barrier to optimal immunosuppression. Nat Rev Immunol 2003; 3:831-838.

29. Kanemaru S, Nakamura T, Yamashita Met al. Destiny of autologous bone marrow-derived stromal cells implanted in the vocal fold. Annals of Otology Rhinology and Laryngology 2005; 114.

30. Chan RW, Gray SD, Titze IR. The importance of hyaluronic acid in vocal fold biomechanics. Otolaryngol Head Neck Surg 2001; 124:607-614.

31. Rousseau B, Hirano S, Scheidt TDet al. Characterization of vocal fold scarring in a canine model. Laryngoscope 2003; 113:620-627.

32. Matsumoto K, Nakamura T. Hepatocyte growth factor (HGF) as a tissue organizer for organogenesis and regeneration. Biochemical and Biophysical Research Communications 1997; 239:639-644.

33. Kumai Y, Kobler JB, Park H, Galindo M, Herrera VL, Zeitels SM. Modulation of vocal fold scar fibroblasts by adipose-derived stem/stromal cells. Laryngoscope 2010; 120:330-337.

34. Kishimoto Y, Hirano S, Kitani Yet al. Chronic vocal fold scar restoration with hepatocyte growth factor hydrogel. Laryngoscope 2010; 120:108-113.

35. Suehiro A, Hirano S, Kishimoto Y, Tateya I, Rousseau B, Ito J. Effects of Basic Fibroblast Growth Factor on Rat Vocal Fold Fibroblasts. Annals of Otology Rhinology and Laryngology 2010; 119:690-696. 
Figures and a Table

Fig. 1

A
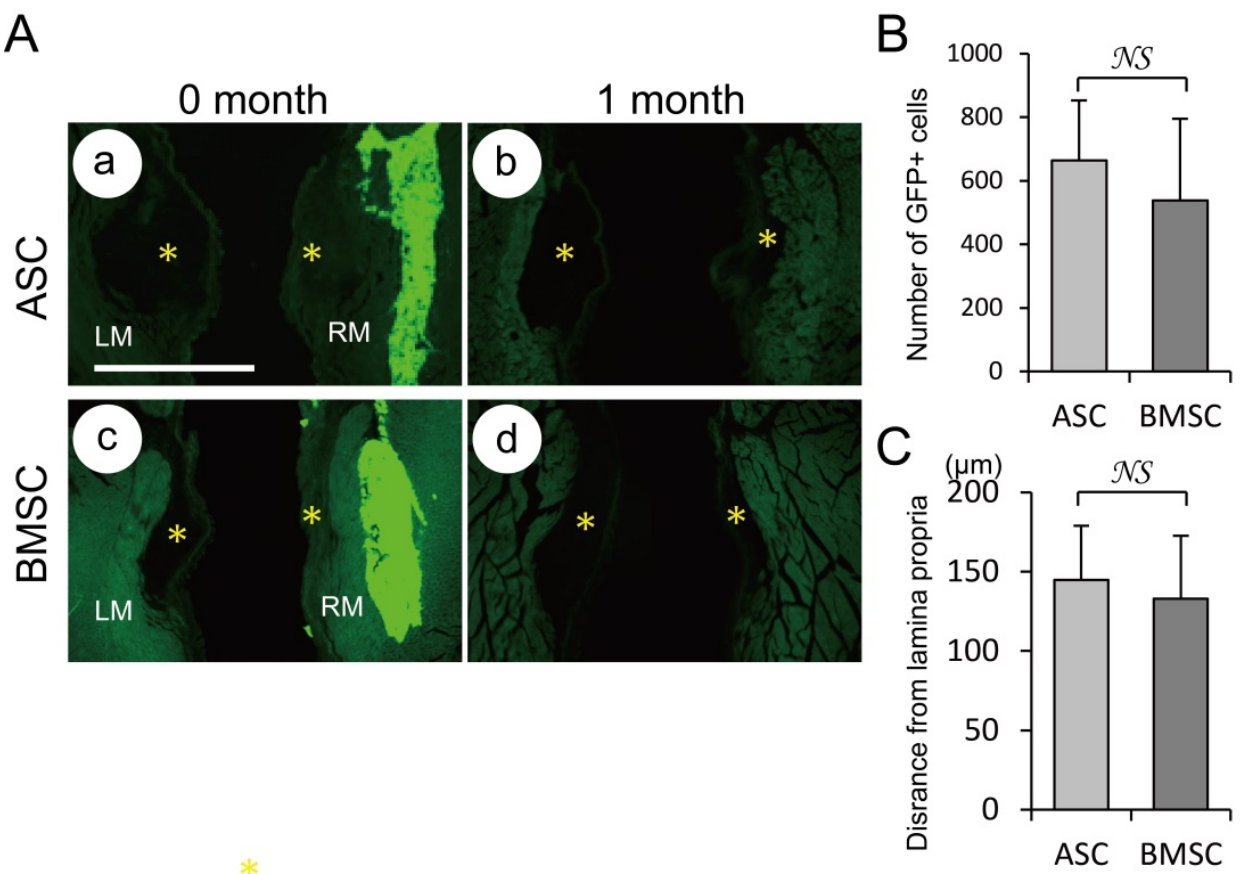
Fig. 2
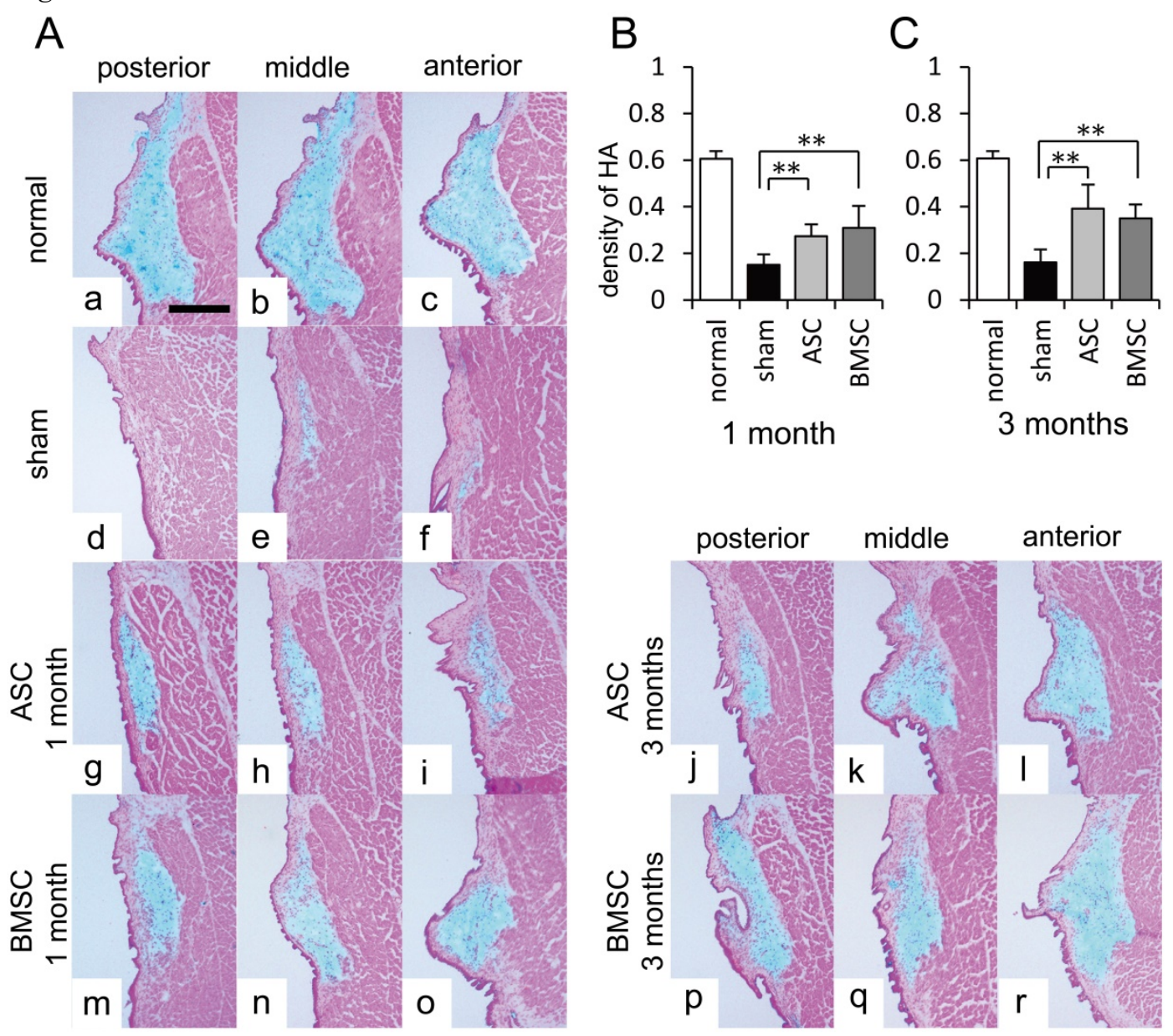
Fig. 3
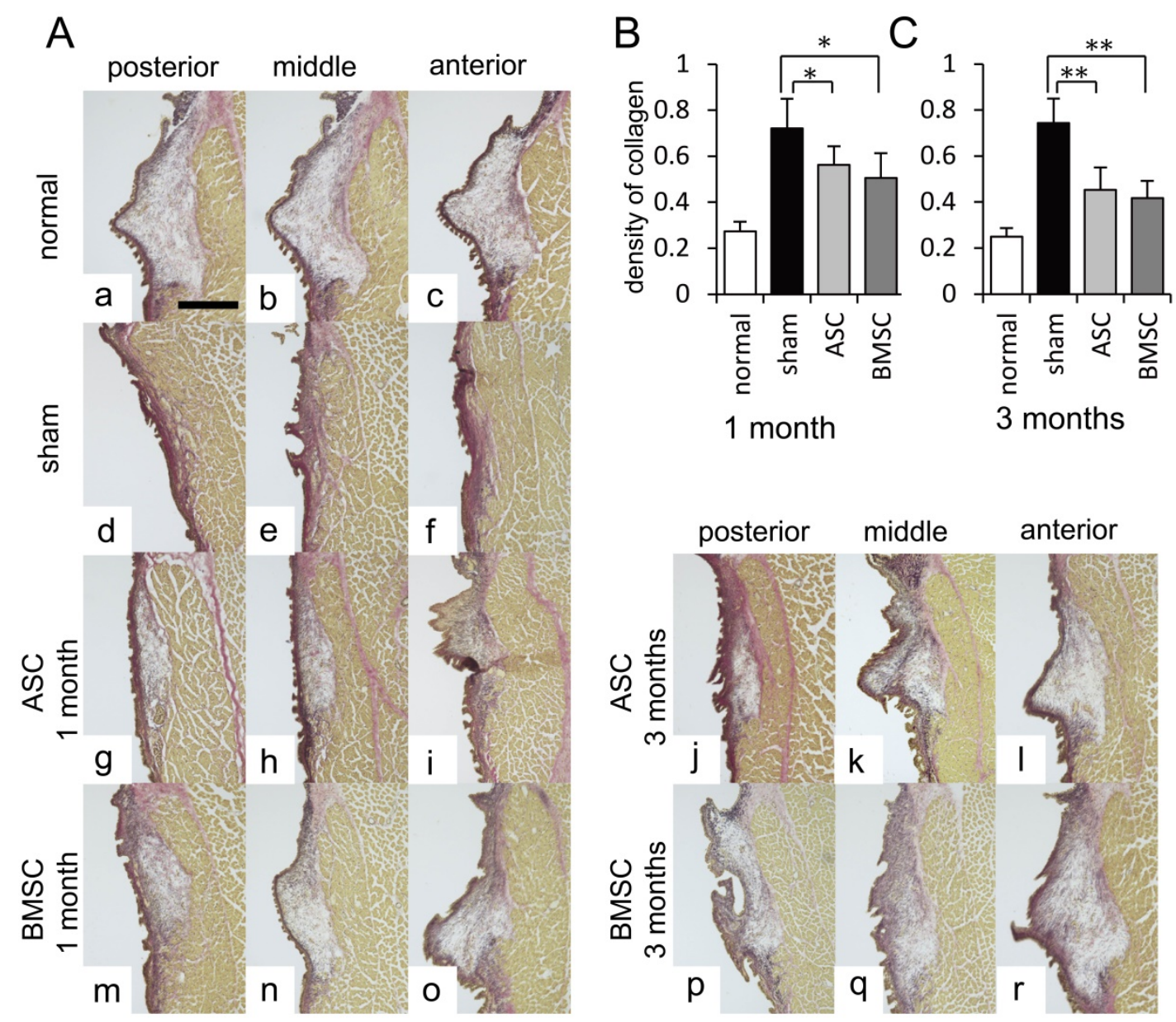
Fig. 4

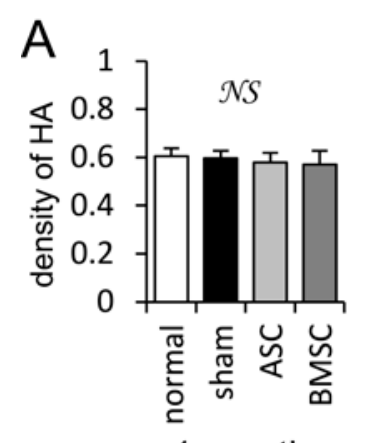

B

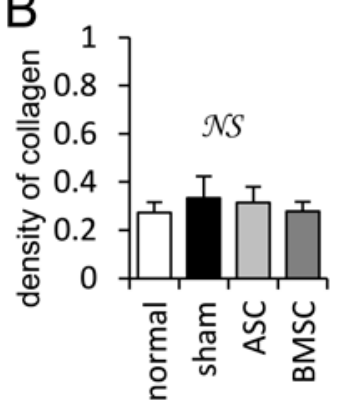

1 month

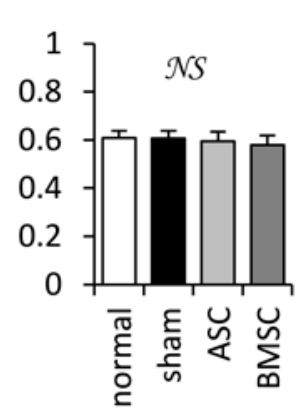

3 months

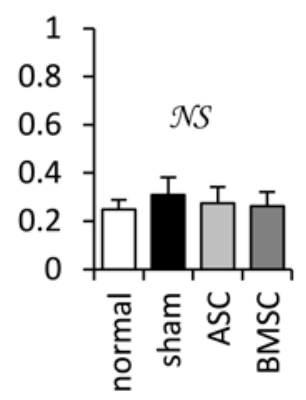

3 months 
Fig. 5
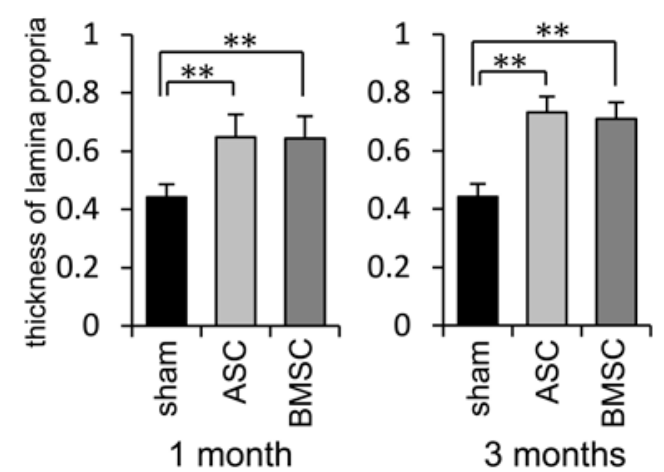

1 month

3 months 
Fig. 6

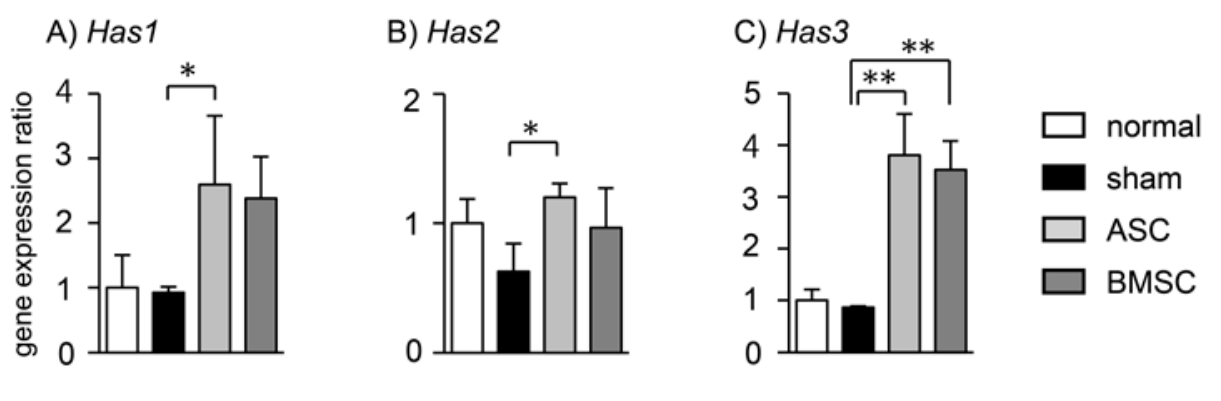
D) Col1a1
E) Co/3a1
F) $M m p 1$
G) $M m p 8$
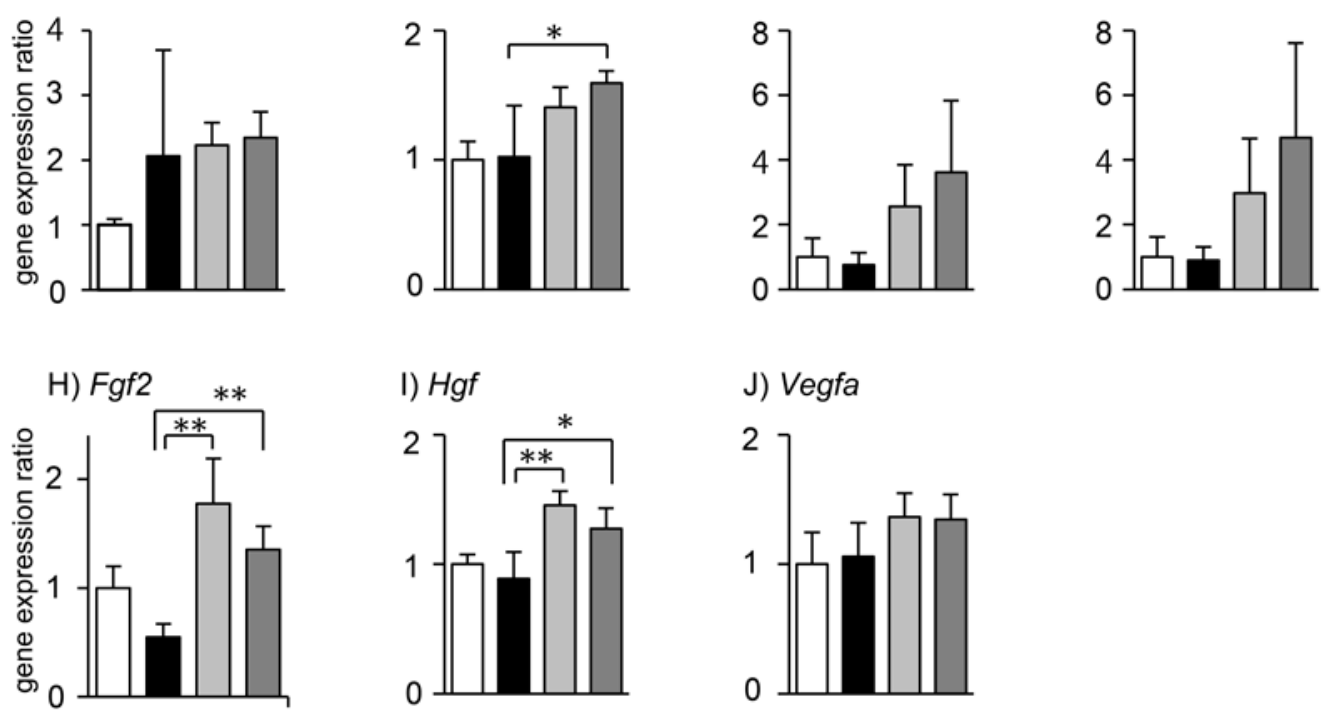

J) Vegfa

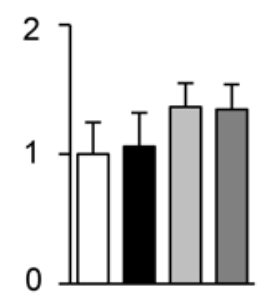


TABLE : Primer Sequences

\begin{tabular}{lll}
\hline Gene & Forward primer & Reverse primer \\
\hline Hyaluronic acid synthase 1 & 5'-TAGGTGCTGTTGGAGGAGATGTGA-3' & 5'-AAGCTCGCTCCACATTGAAGGCTA-3' \\
Hyaluronic acid synthase 2 & 5'-ACTGGGCAGAAGCGTGGATTATGT-3' & 5'-AACACCTCCAACCATCGGGTCTTCTT-3' \\
Hyaluronic acid synthase 3 & 5'-CCTCATCGCCACAGTCATACAA-3' & 5'-CCACCAGCTGCACCGTTAGT-3' \\
Procollagen type I & 5'-AGGCATAAAGGGTCATCGTGGCTT-3' & 5'-AGTCCATCTTTGCCAGGAGAACCA-3' \\
Procollagen type III & 5'-ATGAGCTTTGTGCAATGTGGGACC-3' & 5'-ACTGACCAAGGTAGTTGCATCCCA-3' \\
Matrix metalloproteinase 1 & 5'-GGTGGCCAGAATAGCTGAATG-3' & 5'-GCGTTTTGATATGCCC-3' \\
Matrix metalloproteinase 8 & 5'-CCATGGATCCAGGTTACCCCACT-3' & 5'-TGTGGTCCACTGAAGAAGAGGAAGA-3' \\
Fibroblast growth factor 2 & 5'-AAGAACGGCGGCTTCTTCCT-3' & 5'-CCCTTGATGGACACAACTCC-3' \\
Hepatocyte growth factor & 5'-ACAAGGGCTTTCCATTCACT-3' & 5'-CCAGTAGCATCGTTTTCTCG-3' \\
Vascular endothelial growth factor & 5'-CGTCTACCAGCGCAGCTATTG-3' & 5'0-GTGAGGTTTGATCCGCATGAT-3' \\
B2-microglobulin & 5'-TCACACTGAATTCACACCCACCGA-3' & 5'0-TGATTACATGTCTCGGTCCCAGGT-3' \\
\hline
\end{tabular}


Figure Legends

Fig. 1

(A) Representative immunofluorescent images of GFP (green). (a, b) ASC group, (c, d) BMSC group: (a,c) Month zero, post-transplantation, (b,d) One month post-transplantation. LM: Left thyroarytenoid muscle; RM: Right thyroarytenoid muscle, *: superficial lamina propria. GFP-positive MSCs were located within the outer space of the thyroarytenoid muscle at month 0 , whereas no surviving MSC were detected either in the SLP or in the underlying muscle. Scale bar = $500 \mu \mathrm{m}$. (B) There were no significant differences in the average numbers of GFP-positive cells between the ASC group and the BMSC group at month 0. (C) There were no significant differences between the averaged distances between the lateral edge of LP and the most adjacent transplanted cells in comparisons of the ASC group and the BMSC group at month 0 .

Fig. 2

(A) Representative Alcian blue staining of the normal group (a, b, c), the sham-treated group (d, e, f), the ASC group (g, h, i, 1 month post-transplantation; j, k, l, 3 months post-transplantation), and the BMSC group (m, n, o, 1 month post-transplantation; p, q, r, 3 months post-transplantation). Scale Bar $=200 \mu \mathrm{m} .(\mathrm{B}, \mathrm{C})$ The densities of HA-stained areas were significantly greater in the ASC and BMSC groups than the sham-treated group, whereas there was no significant difference between the 2 transplantation groups. (B) One month post-transplantation, (C) Three months post-transplantation. ${ }^{*} p<0.05,{ }^{* *} p<0.01$, one-way factorial analysis of variance with a post hoc Tukey-Kramer test. Bars represent the standard deviations.

Fig. 3 
(A) Representative Elastica van Gieson staining of collagen in the normal group (a, b, c), the sham-treated group (d, e, f), the ASC group (g, h, i, 1 month post-transplantation; j, k, l, 3 months post-transplantation), and the BMSC group (m, n, o, 1 month post-transplantation; p, q, r, 3 months post-transplantation). Scale Bar $=200 \mu \mathrm{m}$. (B, C) The densities of collagen-abundant areas were significantly decreased in the ASC and BMSC group compared to the sham-treated group, whereas there was no significant difference between the 2 transplantation groups. (B) One month post-transplantation. (C) Three months post-transplantation. ${ }^{*} p<0.05$, ${ }^{* *} p<0.01$, one-way factorial analysis of variance with a post hoc Tukey-Kramer test. Bars represent the standard deviations.

Fig. 4

The densities of HA-stained areas (A) and collagen-abundant areas (B) of contralateral side (left uninjured side). There was no significant difference between each group at both one and three months post-transplantation. One-way factorial analysis of variance with a post hoc Tukey-Kramer test. Bars represent the standard deviations.

Fig. 5

Normalized thicknesses of lamina propria. The normalized thickness of the LP was significantly greater in both the ASC and BMSC groups at 1 and 3 months post-transplantation compared with the sham-treated group, whereas there was no significant difference between the 2 transplantation groups. Bars represent the standard deviation. ${ }^{* *} p<0.01$,Student $t$ test. Bars represent the standard deviations.

Fig. 6

Gene expression ratios 1 month post-transplantation. (A, B) Significant differences were observed 
for Has1 and Has2 in the ASC group vs. the sham-treated group. (E) Significant differences were observed for Col3a1 in the BMSC group vs. sham-treated group. (C, H, I) Significant differences were observed for Has3, Fgf2, Hgf in both the ASC and the BMSC groups vs. the sham-treated group. ${ }^{*} p<0.05,{ }^{* *} p<0.01$, one-way factorial analysis of variance with a post hoc Tukey-Kramer test. Bars represent the standard deviations. 